\title{
Dispar calidad de las guías de práctica clínica para EPOC
}

Critical Appraisal of Clinical Practice Guidelines Targeting Chronic Obstructive Pulmonary Disease. Lacasse Y, Ferreira I, Brooks D, y col. Arch Intern Med. 2001; 161:69-74

\section{Objetivo}

Examinar el contenido y evaluar críticamente las Guías de Práctica Clínica (GPC) existentes para el manejo de la enfermedad pulmonar obstructiva crónica (EPOC).

\section{Metodología}

Los autores evaluaron las GPC recientemente publicadas para el manejo de la EPOC analizando los potenciales orígenes de disparidad entre su contenido. Se buscó en el MEDLINE entre 1990 y 1999 las GPC vinculadas al manejo general de los pacientes con EPOC, usando como palabras clave: 1) enfermedad pulmonar obstructiva; 2) guías. Se hallaron 15 GPC.

Se seleccionó, por otro lado, el mejor de los 12 instrumentos existentes para valorar la calidad científica de una GPC (validez y confiabilidad). Se evaluaron tres dimensiones metodológicas: 1) desarrollo de la GPC; 2) contexto y contenido; 3) diseminación e implementación. Cuatro revisores evaluaron independientemente cada GPC para el manejo de los pacientes con EPOC.

\section{Resultados Principales}

- Ninguna de las GPC cumplió con el criterio primario de validez ya que no estaban completamente basadas en evidencia a través de revisiones sistemáticas de la literatura.

- Todas las GPC incluían una correcta definición de la población a la que estaban dirigidas y una sola de ellas incluía estimaciones del costo asociado al manejo recomendado.

- Sólo dos de las GPC sugerían posibles métodos para su implementación. La claridad de las recomendaciones era escasa, lo que se vio reflejado por el moderado grado de acuerdo entre los revisores (índice kappa medio: 0.41; rango intercuartilo:
0.21 a 0.85).

- Todas las GPC recomiendan dejar de fumar y casi todas recomiendan la vacunación contra Influenza.

- La recomendación de vacunarse contra Pneumococo es variable.

- Otras áreas de controversia incluyen el uso de b2-agonistas vs agentes anticolinérgicos como broncodilatadores de primera línea, la indicación de mucolíticos, el rol de los corticoides inhalatorios y la prescripción de oxigenoterapia para los pacientes que presentan desaturación durante el ejercicio y el sueño. Aún cuando existieron criterios unánimes respecto a una recomendación particular se identificaron discrepancias en la aplicación.

Si bien el desarrollo de las GPC basadas en evidencia representa uno de los mayores cambios en la actitud médica; por un lado, las revisiones sistemáticas de la literatura consumen tiempo y son costosas y, por otro, existe insuficiente cantidad de ensayos clínicos bien dieñados para confeccionar GPC con buen nivel de evidencia científica, lo que obliga a llenar estos huecos con recomendaciones de consensos y opiniones de expertos.

\section{Conclusiones}

El propósito de ésta comunicación no es apoyar estrictamente una estrategia de manejo de los pacientes con EPOC, sino mostrar que las GPC no son completamente basadas en evidencia. Ellas varían en sus recomendaciones específicas, reflejando de ésta forma los sesgos en la experiencia selectiva más que en el conocimiento científico. Finalmente, las GPC deberían incluir conceptos sobre su implementación y evaluación del impacto que producen en sus recomendaciones.

\section{Comentario}

Las GPC son recomendaciones generadas para el cuidado de condiciones médicas específicas y están basadas en la mejor evidencia disponible y en la experiencia práctica. Deben ser interpretadas como sugerencias para el cuidado de los pacientes y no como reglas estrictas ya que el manejo de cada persona debe ser individualizado. Razones legítimas para ello son las diferencias biológicas en el metabolismo de drogas y las respuestas inmunológicas, las distintas comorbilidades de cada individuo, las diferencias sociales y económicas en el nivel local de la práctica clínica, como así tambien, en las preferencias de los pacientes ${ }^{1}$. Las GPC deberían estar basadas en revisiones sistemáticas de investigaciones publicadas, siguiendo una serie de reglas preestablecidas para identificar estudios relevantes y juzgar así la solidez científica, mostrando una absoluta transparencia en la racionalidad de sus decisiones. La opinión de expertos y la práctica usual no soportada por investigaciones puede ser incluída, pero debería ser rotulada como tal y no tomar prioridad sobre evidencia fuerte. Las GPC que versan sobre el mismo tema, realizadas por diferentes grupos de expertos frecuentemente muestran desacuerdos; pero usualmente las diferencias son menores. Este punto puede edificarse como una importante barrera para la aceptación de las guías. Vale aclarar que el desacuerdo entre las GPC no necesariamente es una señal de mala calidad2.

La enfermedad pulmonar obstructiva crónica es la principal causa de morbilidad por una patología crónica y la cuarta causa desencadenante de muerte en el mundo; por lo tanto, se requiere un importante esfuerzo internacional unificado para revertir esta situación. Dos meses después de que se presentara esta publicación, se dieron a conocer los resultados del proyecto colaborativo del U.S. National Heart, Lung and Blood Institute (NHLBI) y de la World Health Organization, el mismo se conoció con el nombre de Global Initiative for Chronic Obstructive Lung Disease (GOLD)3. EI GOLD Workshop reportó la estrategia global para el diagnóstico, manejo y prevención del EPOC, abarcando cuatro componentes: 1) asesorar y monitorear la enfermedad; 2) reducir los factores de riesgo; 3) manejo del EPOC estable; 4) manejo de las exacerbaciones. El reporte se encuentra basado en los conceptos mejor validados al corriente, reunidos por expertos en la patología y en técnicas de investigación. Por otro lado, cada uno de las aseveraciones presentadas en la guía se acompañan de la asignación de evidencias, mediante el uso de un sistema desarrollado por el NHLBI.

\section{Dr. Hernán Michelángelo [ Servicio de Clínica Médica. Hospital Italiano de Buenos Aires ]}

\section{Referencias}

1. Woolf, SH. Practice guidelines: A new reality in medicine. I. Recent developments. Arch Intern Med 1990; 150:1811

2. Woolf, SH. Practice guidelines, a new reality in medicine. II. Methods of developing guidelines. Arch Intern Med 1992; 152:946.

3. Pauwels R, Buist S, Calverley P y col. Global Strategy for the Diagnosis, Management, and Prevention of Chronic Obstructive Pulmonary Disease. NHLBI/WHO Global Initiative for Chro-

nic Obstructive Lung Disease (GOLD). Am J Respir Crit Care Med, Vol 163. Pp 1256-1276, 2001 